\title{
Factores vinculados a la calidad de vida en la adultez mayor
}

\author{
ALFONSO URZÚA ${ }^{\mathrm{a}}$, MIGUEL BRAVO ${ }^{\mathrm{b}}$, \\ MARIO OGALDE $^{\mathrm{b}}$, CAROLINA VARGAS ${ }^{\mathrm{b}}$
}

Escuela de Psicología, Universidad Católica del Norte.

apsicólogo, Licenciado en Psicología, Magíster en Salud Pública, Doctor en

Psicología clínica y de la Salud.

bPsicólogo(a), Licenciado(a) en Psicología.

Recibido el 19 de enero de 2011, aceptado el 1 de junio de 2011.

Correspondencia a: Dr. Alfonso Urzúa Escuela de Psicología Universidad Católica del Norte, Avda. Angamos 0610, Antofagasta, Chile. E-mail: alurzua@ucn.cl

\section{Quality of life of older people living in Antofagasta, Chile}

Background: As basic needs of older people are covered, the concern about the determinants of their quality of life becomes preeminent. Aim: To evaluate the relationship between self-reported quality of life and related variables. Material and Methods: The Quality of Life Survey for older people developed by the World Health Organization (WHOQOL-Old), the reduced scale of Ryff Psychological Well Being, the Functional Social Support Questionnaire, the SF-12 and GHQ12 general health surveys were applied to 406 older adults aged $71 \pm 7$ years ( $83 \%$ women), that were members of older people organizations and lived in Antofagasta, Chile. Results: Older people that perceived themselves as sick had significantly lower quality of life scores. Self-acceptance, social support, autonomy and having a purpose in life also influenced the perception of quality of life. Conclusions: Health issues and the sense of self efficacy are determinants of the quality of life of these older subjects.

(Rev Med Chile 2011; 139: 1006-1014).

Key words: Aged; Quality of life; Questionnaires.
$\mathrm{E}$ n Chile, en el 2025, los adultos mayores (AM) constituirán el $20 \%$ de la población ${ }^{1,2}$, generando nuevas necesidades sociosanitarias. Actualmente, los Estados intentan cubrir estas necesidades desde una perspectiva sanitaria o desde los programas sociales ${ }^{2,3}$. Consecuente con esto, los estudios asociados al adulto mayor (AM) suelen centrarse en indicadores objetivos como el estado de salud ${ }^{4-6}$ o el nivel y condiciones de vida ${ }^{7-10}$, siendo usualmente los resultados utilizados como base para la planificación de políticas públicas y lineamientos en temáticas relevantes para los $\mathrm{AM}^{2,7,11}$.

A fin de ampliar la mirada evaluativa e incluir indicadores comprensivos del bienestar, la Organización Mundial de la Salud llamó a la acción para cambiar las actitudes, políticas y planes en torno a un envejecimiento activo y saludable ${ }^{2,12-14}$. Es aquí donde la evaluación de la Calidad de Vida (CV) toma relevancia como indicador en la planificación de políticas públicas para los $\mathrm{AM}^{7,11,13}$, siendo definida como la percepción del individuo de su posición en la vida, en el contexto de la cultura y el sistema de valores en los cuales vive, en relación con sus objetivos, expectativas, categorías y preocupaciones ${ }^{15}$.

Diversos factores y variables biopsicosociales se han relacionado con la CV del AM, entre estos el bienestar psicológico ${ }^{14,16,17}$, apoyo social ${ }^{5,18,19}$, estado de salud en general ${ }^{10}$ y estado de salud mental y física $^{5}$. En Chile, sólo se ha encontrado referencias a un estudio vinculado con la situación de las viviendas, aun cuando no se utilizó un instrumento específico para este grupo etario?.

Dado que la evaluación que una persona hace de su CV incorpora elementos socio-culturales y valóricos propios del contexto en que vive en $^{11,20-22}$ es de interés saber si existirán diferencias entre factores relacionados con CV en el AM entre los distintos países y aun más relevante es saber cuáles son los factores vinculados a la CV en nuestro país. Bajo este contexto, esta investigación tiene por 
objetivo analizar la CV desde la perspectiva de las personas mayores y su relación con diversas variables teóricas y empíricamente relacionadas con $\mathrm{CV}$ como el bienestar psicológico, apoyo social, estado general de salud y estado físico y mental de salud.

\section{Pacientes y Método}

\section{Participantes}

El muestreo fue no probabilístico y por conveniencia. Se repartieron 600 cuestionarios a AM pertenecientes a agrupaciones gubernamentales $y$ no gubernamentales de la ciudad de Antofagasta, Chile, retornando 450 cuestionarios. Cuarenta y cuatro cuestionarios fueron eliminados por incompletos. La muestra final estuvo constituida por 406 participantes, de los cuales 359 pertenecían a organizaciones sociales $(88,4 \%)$ y 47 AM contactados por redes informales $(11,6 \%)$. Trescientos treinta y cinco eran mujeres $(82,5 \%)$ y 71 hombres (17,5\%). La edad osciló entre los 60 y los 90 años, con una media de 71 años $(\mathrm{DE}=6,7)$ en la muestra total, 71,05 años $(\mathrm{DE}=6,78)$ en las mujeres y 70,76 años $(\mathrm{DE}=6,59)$ en los hombres. El 76,1\% de los evaluados reportaron presencia de enfermedades diagnosticadas, siendo las más frecuentes la hipertensión arterial (19\%), diabetes mellitus (9,9\%) y las musculoesqueléticas $(9,6 \%)$. Independientemente de la presencia de una enfermedad diagnosticada, $38,4 \%$ de las personas perciben sentirse enfermas, mientras que $57,4 \%$ no se sentía enfermo.

\section{Instrumentos}

La CV se evaluó a través del WHOQOL- Old, diseñado por la Organización Mundial de la Salud específicamente para AM. Está compuesto por
24 ítems agrupados en 6 dominios (Tabla 1), con formato de respuesta tipo Likert con 5 opciones, donde un mayor puntaje representa una mayor $\mathrm{CV}^{21}$. Se ha reportado características psicométricas adecuadas en población brasileña ( $\alpha$ de Cronbach $0,71$ a 0,88$)^{20}$. En nuestro estudio se obtuvo un $\alpha$ de Cronbach 0,83.

Para evaluar los diversos factores se utilizó la versión de 29 ítems de la Escala de Bienestar Psicológico de Ryff ${ }^{23}$, la Escala de Apoyo Social -Duke-UNC ${ }^{24}$, el cuestionario de salud física y mental SF- $12^{25}$ y el Cuestionario general de salud GHQ- $12^{26}$.

\section{Procedimientos}

Una vez aprobado el proyecto por el Comité de Ética de la Universidad Católica del Norte, se contactó a la totalidad de representantes de agrupaciones de AM pertenecientes a la red del Servicio Nacional del Adulto Mayor, a fin de gestionar la participación de sus integrantes. En cada asamblea se explicó el objetivo de la investigación. Cada persona que accedió a participar firmó un acta de consentimiento informado. Una vez entregadas las instrucciones para completar el cuestionario, se indicó un plazo de una semana para contestarlo. Transcurrido dicho período, los investigadores volvieron a cada lugar de reunión para recoger aquellas encuestas que habían sido contestadas.

Una vez ingresados los datos, se realizaron análisis descriptivos de las variables, pruebas de diferencias de medias según sexo, edad, presencia de enfermedad, percepción de enfermedad y participación en organizaciones sociales (pruebas T y Anova de un Factor). A fin de analizar la relación entre las variables se utilizó el coeficiente de correlación de Pearson. Finalmente, se realizaron

Tabla 1. Dominios de calidad de vida evaluados por el WHOQOL-Old

\begin{tabular}{|c|c|}
\hline Dominio & Definición \\
\hline Habilidades sensoriales & Impedimento sensorial que afecta la vida diaria y el funcionamiento general \\
\hline Autonomía & $\begin{array}{l}\text { Capacidad para tomar decisiones propias, sentirse en control del propio fu- } \\
\text { turo y de hacer las cosas que se quiere }\end{array}$ \\
\hline Actividades del pasado, presente y futuras & $\begin{array}{l}\text { Grado de satisfacción con los logros pasados y futuros y con el reconocimien- } \\
\text { to de lo realizado }\end{array}$ \\
\hline Participación social & Grado de satisfacción en las distintas actividades que participa \\
\hline Muerte y el morir & Grado de preocupación hacia la muerte y el morir \\
\hline Intimidad & Oportunidad para amar y ser amado \\
\hline
\end{tabular}


regresiones lineales considerando como variables dependientes la $\mathrm{CV}$ y sus respectivos dominios y como variables independientes bienestar psicológico, apoyo social, salud física y mental, y estado de salud.

\section{Resultados}

En todos los grupos, el dominio mejor evaluado fue el de participación social y el peor el de la muerte y el morir (Tabla 2).

Al comparar por sexo, la única diferencia estadísticamente significativa estuvo en el dominio de participación social $\left(\mathrm{t}_{(87,80)}=2,24\right.$, $\mathrm{p}<, 05 ; \mathrm{r}=, 23$ ), donde la media de las mujeres es mayor que la de los hombres.

$\mathrm{Al}$ analizar por presencia de enfermedad, no se encontraron diferencias estadísticamente significativas en las medias de los dominios entre ambos grupos. Al estratificar por percepción de enfermedad, se encontraron diferencias estadísticamente significativas entre ambos grupos en todos los dominios evaluados, siendo mayor la media obtenida por el grupo que no se siente enfermo en los dominios de autonomía $\left(\mathrm{t}_{(387)}=-2,62, \mathrm{p}<, 01 ; \mathrm{r}=, 13\right)$, actividades del pasado, presente y futuro $\left(\mathrm{t}_{(263.842)}=-5,10\right.$, $\mathrm{p}<, 01 ; \mathrm{r}=, 30) ;$ participación social $\left(\mathrm{t}_{(282,359)}=-5,08, \mathrm{p}<, 01 ; \mathrm{r}=, 29\right)$, intimidad $\left(\mathrm{t}_{(387)}=-4,71, \mathrm{p}<, 01 ; \mathrm{r}=, 23\right)$ y a nivel de la evaluación global de la CV $\left(\mathrm{t}_{(275,332)}=-2,83\right.$, $\mathrm{p}<, 05 ; \mathrm{r}=, 17)$. El grupo que se percibe como enfermo tuvo una media significativamente mayor que el que no en los dominios de habilidades sensoriales $\left(\mathrm{t}_{(387)}=2,57, \mathrm{p}<, 05\right.$; $\mathrm{r}=, 13)$ y muerte y el morir $\left(\mathrm{t}_{(303,394)}=3,46\right.$, $\mathrm{p}<, 05 ; \mathrm{r}=, 19)$.

Al estratificar por participación en agrupaciones o no, sólo se evidencia diferencia significativa en el dominio Participación social $\left(\mathrm{t}_{(60.833)}=3,27, \mathrm{p}<, 05 ; \mathrm{r}=, 01\right)$.

$\mathrm{Al}$ relacionar la edad de los participantes con el puntaje obtenido en los diversos dominios, el único que correlacionó de manera significativa e inversa fue muerte y el morir $\left(\mathrm{r}=-108, \mathrm{p}<, 05 ; \mathrm{r}^{2}=, 04\right)$.

Considerando como variable dependiente la evaluación de la CV general, las dimensiones de autoaceptación, apoyo social

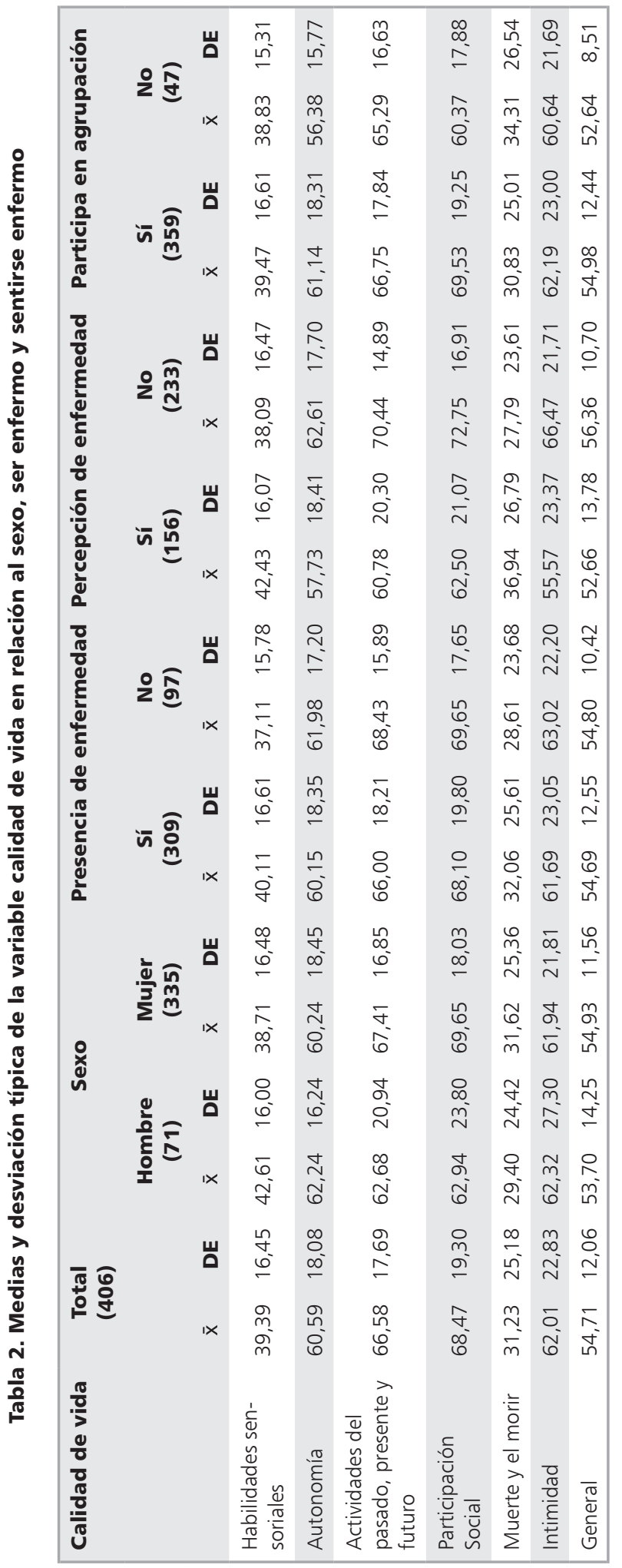


y autonomía, contribuyen de forma significativa al modelo $\left(\mathrm{F}_{(93242)}=8,06 \mathrm{E} 03, \mathrm{p}<, 01\right)$, siendo capaces de explicar $41 \%$ de la varianza de la variable (Tabla 3). Para esta misma variable, fueron significativas al modelo en la submuestra de personas que se sienten enfermas (Tabla 4), las dimensiones auto aceptación y apoyo social, (49\% de la varianza). En el grupo de personas que no se sienten enfermas, las dimensiones significativas fueron el propósito en la vida y el apoyo social (33\% de la varianza) (Tabla 5).

Al considerar como variable dependiente el dominio de CV habilidades sensoriales, en la muestra total (Tabla 3), fueron significativas al modelo las dimensiones de autonomía, autoaceptación y salud física $\left(\mathrm{F}_{(16.403)}=3,98 \mathrm{E}+03 ; \mathrm{p}<, 01\right)$, las que explican $11 \%$ la varianza de la variable. En el grupo de personas que se sienten enfermas, sólo fueron significativas al modelo la autoaceptación y la autonomía (Tabla 4), en tanto en el grupo de personas que no se sienten enfermas fueron la autonomía y la salud física (Tabla 5).

$\mathrm{Al}$ considerar como variable dependiente el dominio de CV autonomía, en la muestra total el modelo incorporó las dimensiones propósito $\mathrm{y}$ apoyo social $\left(\mathrm{F}_{(64,6522)}=1,61 \mathrm{E}+04, \mathrm{p}<, 01\right)$, siendo éstas capaces de explicar 24\% la varianza del dominio. En el grupo de personas que se sienten enfermas, sólo fue significativa la variable autoaceptación, la que explicó el 31\% la varianza de la variable autonomía. En el grupo de personas que no se sienten enfermas, fueron significativas al modelo las dimensiones propósito en la vida y autonomía (21\% de la varianza).

Considerando como variable dependiente el dominio de CV actividades del pasado, presente

Tabla 3. Análisis de regresión para variable dependiente calidad de vida y sus dominios

\begin{tabular}{|c|c|c|c|c|c|c|}
\hline \multirow[t]{2}{*}{$\begin{array}{l}\text { Variables } \\
\text { dependientes }\end{array}$} & \multirow[t]{2}{*}{ Modelo } & \multicolumn{2}{|c|}{$\begin{array}{l}\text { Coeficiente no } \\
\text { estandarizados }\end{array}$} & \multicolumn{2}{|c|}{$\begin{array}{c}\text { Coeficiente } \\
\text { estandarizados }\end{array}$} & \multirow[b]{2}{*}{ Sig. } \\
\hline & & $\boldsymbol{\beta}$ & Error típ. & $\boldsymbol{\beta}$ & $\mathbf{t}$ & \\
\hline Calidad de vida general & $\begin{array}{l}\text { (Constante) } \\
\text { Apoyo social } \\
\text { Auto aceptación } \\
\text { Autonomía }\end{array}$ & $\begin{array}{r}21,212 \\
0,327 \\
6,296 \\
-2,142\end{array}$ & $\begin{array}{l}3,153 \\
0,052 \\
0,590 \\
0,651\end{array}$ & $\begin{array}{r}0,278 \\
0,482 \\
-0,131\end{array}$ & $\begin{array}{r}6,726 \\
6,294 \\
10,679 \\
-3,292\end{array}$ & $\begin{array}{l}0,000 \\
0,000 \\
0,000 \\
0,001\end{array}$ \\
\hline Habilidades sensoriales & $\begin{array}{l}\text { (Constante) } \\
\text { Salud física } \\
\text { Auto aceptación } \\
\text { Autonomía }\end{array}$ & $\begin{array}{r}61,564 \\
-0,421 \\
3,821 \\
-5,267\end{array}$ & $\begin{array}{l}6,030 \\
0,099 \\
0,892 \\
1,091\end{array}$ & $\begin{array}{r}-0,207 \\
0,214 \\
-0,237\end{array}$ & $\begin{array}{r}10,210 \\
-4,259 \\
4,284 \\
-4,825\end{array}$ & $\begin{array}{l}0,000 \\
0,000 \\
0,000 \\
0,000\end{array}$ \\
\hline Autonomía & $\begin{array}{l}\text { (Constante) } \\
\text { Apoyo social } \\
\text { Propósito en la vida }\end{array}$ & $\begin{array}{r}15,042 \\
0,219 \\
7,968\end{array}$ & $\begin{array}{l}4,120 \\
0,088 \\
0,951\end{array}$ & $\begin{array}{l}0,124 \\
0,419\end{array}$ & $\begin{array}{l}3,651 \\
2,492 \\
8,380\end{array}$ & $\begin{array}{l}0,000 \\
0,013 \\
0,000\end{array}$ \\
\hline $\begin{array}{l}\text { Actividades del presente, } \\
\text { pasado y futuro }\end{array}$ & $\begin{array}{l}\text { (Constante) } \\
\text { Salud mental } \\
\text { Apoyo social } \\
\text { Auto aceptación }\end{array}$ & $\begin{array}{r}-8,221 \\
0,217 \\
0,463 \\
9,801\end{array}$ & $\begin{array}{l}4,631 \\
0,100 \\
0,069 \\
0,797\end{array}$ & $\begin{array}{l}0,084 \\
0,268 \\
0,511\end{array}$ & $\begin{array}{r}-1,775 \\
2,166 \\
6,709 \\
12,296\end{array}$ & $\begin{array}{l}0,077 \\
0,031 \\
0,000 \\
0,000\end{array}$ \\
\hline Participación social & $\begin{array}{l}\text { (Constante) } \\
\text { Salud física } \\
\text { Apoyo social } \\
\text { Auto aceptación }\end{array}$ & $\begin{array}{r}-3,949 \\
0,258 \\
0,371 \\
10,026\end{array}$ & $\begin{array}{l}5,021 \\
0,095 \\
0,084 \\
0,933\end{array}$ & $\begin{array}{l}0,108 \\
0,197 \\
0,480\end{array}$ & $\begin{array}{r}-0,787 \\
2,713 \\
4,430 \\
10,745\end{array}$ & $\begin{array}{l}0,432 \\
0,007 \\
0,000 \\
0,000\end{array}$ \\
\hline Muerte y el morir & $\begin{array}{l}\text { (Constante) } \\
\text { Salud física } \\
\text { Salud mental } \\
\text { Autonomía }\end{array}$ & $\begin{array}{r}103,222 \\
-0,380 \\
-0,627 \\
-5,891\end{array}$ & $\begin{array}{r}11,033 \\
0,150 \\
0,181 \\
1,671\end{array}$ & $\begin{array}{l}-0,122 \\
-0,171 \\
-0,173\end{array}$ & $\begin{array}{r}9,356 \\
-2,545 \\
-3,473 \\
-3,525\end{array}$ & $\begin{array}{l}0,000 \\
0,011 \\
0,001 \\
0,000\end{array}$ \\
\hline Intimidad & $\begin{array}{l}\text { (Constante) } \\
\text { Apoyo social } \\
\text { Auto aceptación }\end{array}$ & $\begin{array}{r}-14,309 \\
0,867 \\
8,930\end{array}$ & $\begin{array}{l}4,645 \\
0,097 \\
1,078\end{array}$ & $\begin{array}{l}0,390 \\
0,361\end{array}$ & $\begin{array}{r}-3,081 \\
8,941 \\
8,283\end{array}$ & $\begin{array}{l}0,002 \\
0,000 \\
0,000\end{array}$ \\
\hline
\end{tabular}


Tabla 4. Análisis de regresión para sub muestra que se percibe como enferma según variable dependiente calidad de vida y sus dominios

\begin{tabular}{|llccccc|}
\hline $\begin{array}{l}\text { Variables depen- } \\
\text { dientes }\end{array}$ & Modelo & \multicolumn{2}{c}{$\begin{array}{c}\text { Coeficiente } \\
\text { no estandarizados }\end{array}$} & \multicolumn{2}{c|}{$\begin{array}{c}\text { Coeficiente } \\
\text { estandarizados }\end{array}$} \\
& & $\boldsymbol{\beta}$ & Error típ. & $\boldsymbol{\beta}$ & t & Sig. \\
\hline Calidad de vida general & (Constante) & 8,843 & 3,726 & & 2,373 & 0,019 \\
& Apoyo social & 0,333 & 0,089 & 0,254 & 3,722 & 0,000 \\
& Auto aceptación & 7,338 & 0,945 & 0,530 & 7,768 & 0,000 \\
Habilidades sensoriales & (Constante) & 37,626 & 7,635 & & 4,928 & 0,000 \\
& Auto aceptación & 6,072 & 1,215 & 0,376 & 4,999 & 0,000 \\
& Autonomía & $-5,335$ & 1,643 & $-0,244$ & $-3,246$ & 0,001 \\
Autonomía & (Constante) & 13,623 & 5,450 & & 2,500 & 0,013 \\
& Auto aceptación & 10,297 & 1,239 & 0,556 & 8,308 & 0,000 \\
Actividades del presente, & (Constante) & $-7,769$ & 5,146 & & $-1,510$ & 0,133 \\
pasado y futuro & Apoyo social & 0,592 & 0,124 & 0,306 & 4,790 & 0,000 \\
& Auto aceptación & 10,864 & 1,305 & 0,532 & 8,327 & 0,000 \\
Participación social & (Constante) & $-46,404$ & 11,947 & & $-3,884$ & 0,000 \\
& Salud física & 0,631 & 0,164 & 0,227 & 3,843 & 0,000 \\
& Salud mental & 0,702 & 0,198 & 0,224 & 3,553 & 0,001 \\
& Auto aceptación & 12,034 & 1,309 & 0,568 & 9,193 & 0,000 \\
\hline Intimidad & (Constante) & $-14,511$ & 6,589 & & $-2,202$ & 0,029 \\
& Apoyo social & 0,804 & 0,158 & 0,361 & 5,078 & 0,000 \\
& Auto aceptación & 9,386 & 1,671 & 0,399 & 5,619 & 0,000 \\
\hline
\end{tabular}

y futuro, fueron significativas en el modelo las variables autoaceptación, salud mental y apoyo social $\left(\mathrm{F}_{(151.579)}=2,24 \mathrm{E}+04, \mathrm{p}<, 01\right)$, las que explican un $53 \%$ de la varianza de la variable. En el grupo de personas que se sienten enfermas, sólo fueron significativas al modelo la dimensión autoaceptación y apoyo social (55\% la varianza), en tanto en las personas que no se sienten enfermas, lo fueron las dimensiones autoaceptación, propósito en la vida, apoyo social y salud mental (50\% de la varianza).

$\mathrm{Al}$ analizar tomando como variable dependiente el dominio de CV participación social, en la muestra total fueron significativas en el modelo las variables salud física, apoyo social y autoaceptación $\left(\mathrm{F}_{(92.246)}=2,05 \mathrm{E}+04, \mathrm{p}<, 01\right)$, explicando $41 \%$ de la varianza del dominio participación social (Tabla 3). Para la submuestra de personas que sienten enfermas, se observaron variaciones en las dimensiones, siendo sólo significativas la autoaceptación, la salud física y la salud mental (Tabla 4). En la submuestra de personas que no se sienten enfermas sólo fueron significativas al modelo la autoaceptación y el apoyo social (Tabla 5).

En la muestra total, y al considerar como varia- ble dependiente la dimensión muerte y el morir, fueron significativas al modelo la salud física, salud mental y autonomía $\left(\mathrm{F}_{(14.833)}=8,53 \mathrm{E}+03\right.$, $\mathrm{p}<0,01$ ), las cuales explican el $10 \%$ la varianza de dicha dimensión. En las personas que se sienten enfermas ninguna variable fue significativa al modelo, en tanto que en las personas que no se sienten enfermas si lo fueron la autoaceptación, el propósito en la vida y la salud mental (Tabla 5).

Por último, al considerar como variable dependiente el dominio de la CV intimidad, fueron variables significativas al modelo las variables autoaceptación y apoyo social $\left(\mathrm{F}_{(146.763)}=4,45 \mathrm{E}+04\right)$, $\mathrm{p}<0,01$ ), las cuales fueron capaces de explicar en un $42 \%$ la varianza de ese dominio en la muestra total.

\section{Discusión}

El dominio peor evaluado por todos los participantes fue el de la muerte y el morir, en donde a medida que aumentan los años, aumenta el miedo a la muerte. A diferencia de otros estudios, la muerte no sería percibida como una etapa na- 
Tabla 5. Análisis de regresión para sub muestra que no se percibe como enferma según variable dependiente calidad de vida y sus dominios

\begin{tabular}{|c|c|c|c|c|c|c|}
\hline \multirow[t]{2}{*}{$\begin{array}{l}\text { Variables } \\
\text { dependientes }\end{array}$} & \multirow[t]{2}{*}{ Modelo } & \multicolumn{2}{|c|}{$\begin{array}{l}\text { Coeficiente no estan- } \\
\text { darizados }\end{array}$} & \multicolumn{2}{|c|}{$\begin{array}{l}\text { Coeficiente estanda- } \\
\text { rizados }\end{array}$} & \multirow[b]{2}{*}{ Sig. } \\
\hline & & $\boldsymbol{\beta}$ & Error típ. & $\boldsymbol{\beta}$ & $\mathbf{t}$ & \\
\hline Calidad de vida general & $\begin{array}{l}\text { (Constante) } \\
\text { Apoyo social } \\
\text { Propósito en la vida }\end{array}$ & $\begin{array}{r}19,900 \\
0,290 \\
5,016\end{array}$ & $\begin{array}{l}3,455 \\
0,067 \\
0,727\end{array}$ & $\begin{array}{l}0,260 \\
0,413\end{array}$ & $\begin{array}{l}5,759 \\
4,341 \\
6,903\end{array}$ & $\begin{array}{l}0,000 \\
0,000 \\
0,000\end{array}$ \\
\hline Habilidades sensoriales & $\begin{array}{l}\text { (Constante) } \\
\text { Autonomía } \\
\text { Salud física }\end{array}$ & $\begin{array}{l}74,578 \\
-4,567 \\
-0,378\end{array}$ & $\begin{array}{l}8,820 \\
1,401 \\
0,153\end{array}$ & $\begin{array}{l}-0,208 \\
-0,157\end{array}$ & $\begin{array}{r}8,456 \\
-3,260 \\
-2,465\end{array}$ & $\begin{array}{l}0,000 \\
0,001 \\
0,014\end{array}$ \\
\hline Autonomía & $\begin{array}{l}\text { (Constante) } \\
\text { Autonomía } \\
\text { Propósito en la vida }\end{array}$ & $\begin{array}{r}31,149 \\
-3,292 \\
9,393\end{array}$ & $\begin{array}{l}7,315 \\
1,431 \\
1,216\end{array}$ & $\begin{array}{r}-0,139 \\
0,468\end{array}$ & $\begin{array}{r}4,258 \\
-2,301 \\
7,723\end{array}$ & $\begin{array}{l}0,000 \\
0,022 \\
0,000\end{array}$ \\
\hline $\begin{array}{l}\text { Actividades del presente, } \\
\text { pasado y futuro }\end{array}$ & $\begin{array}{l}\text { (Constante) } \\
\text { Apoyo social } \\
\text { Auto aceptación } \\
\text { Salud mental } \\
\text { Propósito en la vida }\end{array}$ & $\begin{array}{r}-6,000 \\
0,339 \\
5,159 \\
0,343 \\
4,039\end{array}$ & $\begin{array}{l}6,109 \\
0,082 \\
1,673 \\
0,124 \\
1,555\end{array}$ & $\begin{array}{l}0,218 \\
0,285 \\
0,146 \\
0,239\end{array}$ & $\begin{array}{r}-0,982 \\
4,143 \\
3,083 \\
2,768 \\
2,597\end{array}$ & $\begin{array}{l}0,327 \\
0,000 \\
0,002 \\
0,006 \\
0,010\end{array}$ \\
\hline Participación social & $\begin{array}{l}\text { (Constante) } \\
\text { Auto aceptación } \\
\text { Apoyo social }\end{array}$ & $\begin{array}{r}13,894 \\
8,757 \\
0,393\end{array}$ & $\begin{array}{l}5,931 \\
1,230 \\
0,106\end{array}$ & $\begin{array}{l}0,426 \\
0,222\end{array}$ & $\begin{array}{l}2,343 \\
7,118 \\
3,718\end{array}$ & $\begin{array}{l}0,020 \\
0,000 \\
0,000\end{array}$ \\
\hline Muerte y el morir & $\begin{array}{l}\text { (Constante) } \\
\text { Salud mental } \\
\text { Auto aceptación } \\
\text { propósito en la vida }\end{array}$ & $\begin{array}{r}80,536 \\
-0,889 \\
-7,975 \\
6,584\end{array}$ & $\begin{array}{r}12,900 \\
0,262 \\
3,572 \\
3,247\end{array}$ & $\begin{array}{r}-0,238 \\
-0,277 \\
0,246\end{array}$ & $\begin{array}{r}6,243 \\
-3,396 \\
-2,233 \\
2,028\end{array}$ & $\begin{array}{l}0,000 \\
0,001 \\
0,027 \\
0,044\end{array}$ \\
\hline Intimidad & $\begin{array}{l}\text { (Constante) } \\
\text { Apoyo social } \\
\text { Auto aceptación }\end{array}$ & $\begin{array}{r}-12,718 \\
0,914 \\
8,376\end{array}$ & $\begin{array}{l}7,286 \\
0,130 \\
1,512\end{array}$ & $\begin{array}{l}0,403 \\
0,317\end{array}$ & $\begin{array}{r}-1,745 \\
7,043 \\
5,542\end{array}$ & $\begin{array}{l}0,082 \\
0,000 \\
0,000\end{array}$ \\
\hline
\end{tabular}

tural de la vida ${ }^{10}$, existiendo preocupación por la forma en que ellos creen van a morir, de sentirse incapaces de controlarla y miedo a sentir dolor antes de morir ${ }^{21}$.

El dominio mejor evaluado fue el de la participación social. La relevancia de este dominio puede estar dada por la importancia del sentirse satisfecho con el modo de usar su tiempo, su nivel de actividad y con su oportunidad de participar en la comunidad ${ }^{21}$. La participación contribuye a mejorar la CV debido a que disminuye la dependencia del $\mathrm{AM}^{10}$. Las mujeres tienen mayor participación y relaciones sociales más fuertes que los hombres ${ }^{27}$, esto manifiesta la importancia de reforzar la participación en los hombres, no sólo porque estadísticamente poseen un porcentaje menor de participación en organizaciones socia$\mathrm{les}^{2}$, sino también porque la baja participación podría declinar sus habilidades funcionales ${ }^{27}$. Por otro lado, existen referencias que indican que una mayor participación social en mujeres sobre 65 años favorece una mejor salud mental ${ }^{28}$.

Se suma evidencia a que no es tan sólo el estado real de salud de una persona lo que condiciona su evaluación de la CV, sino la percepción que ella tiene sobre si se siente enferma o no ${ }^{29}$. En el AM que se percibe como enfermo, la CV se relaciona con la autoaceptación, el apoyo social, la autonomía y la salud física y mental, en tanto que en los que no, con el propósito en la vida, el apoyo social, la salud mental, la autonomía y la salud física. Es importante recordar que la percepción de enfermedad en este estudio es un factor significativo a todos los aspectos de la CV en el AM, hecho reportado en diferentes países, toda vez que la percepción de enfermedad en el AM se ve influenciada por factores de tipo educativos, socioeconómicos y culturales ${ }^{30}$. 
Sobre la Autoaceptación, en la medida que los AM valoran sus capacidades, habilidades y aceptan sus limitaciones, mejorarían su capacidad para solucionar los problemas de la vida diaria, así como el sentirse satisfecho con las actividades que realiza y relacionarse de forma íntima con los demás. Esto facilitaría alcanzar metas u objetivos, permitiendo adaptarse a su etapa del ciclo vital ${ }^{31-35}$.

En el AM que se perciben como enfermo, el desarrollar una evaluación positiva de sí mismo conllevaría a un mayor control sobre diversos ámbitos de su vida ${ }^{36,37}$.

En esta investigación, para el AM que no se percibe enfermo, la autoaceptación, sería menos relevante para evaluar su $\mathrm{CV}$, enfocándose en establecer metas y propósitos para su vida, y así poder trascender o dejar un legado.

Se ha reportado que a partir de la jubilación, tiende a ser relevante la pérdida del poder, de la autonomía y del rol que ocupaba en la sociedad, surgiendo la necesidad de elaborar un plan de vida que le proporcione satisfacciones y que le permita seguir participando activamente en la sociedad, con el objetivo de que este evento no resulte impactante en su vida ${ }^{34}$.

De igual manera, el Apoyo Social se relacionó directamente con la $\mathrm{CV}$, posiblemente por que el estar integrado en la familia, la comunidad y mantener relaciones sociales activas, puede llevar a un sentimiento de satisfacción por cumplir papeles sociales importantes, $\mathrm{o}$ a sentimientos de autoeficacia, control y competencia personal ${ }^{38,39}, \mathrm{o}$ bien dado que el contar con soporte social podría amortiguar las posibles situaciones estresantes, producto del deterioro de sus capacidades ${ }^{40,41}$.

En los AM que se perciben enfermos, el apoyo social tendría efectos favorables sobre la salud y el bienestar, así como en la disminución de la vulnerabilidad a enfermar. El AM al establecer contactos sociales, se siente más satisfecho consigo mismo, generando una evaluación positiva de su salud física, la cual estaría relacionada con una menor preocupación frente al proceso natural de la muerte ${ }^{42,43}$.

En los AM que no se sienten enfermos, contar con apoyo social le permite enfrentar los retos del ambiente, favoreciendo en cierta medida las posibilidades de plantearse objetivos en la vida ${ }^{41,44}$.

La Autonomía es relevante en la evaluación que el AM hace de su CV, generando un mayor control sobre sus capacidades y habilidades, provocando una adecuada adaptación al entorno ${ }^{45}$.
El Propósito en la vida está asociado a la CV de las personas que no se perciben enfermas, posiblemente este posibilitaría una adaptación positiva a esta etapa, y permitiría recuperar aspectos que proporcionan una vida más plena, rescatando el derecho a sentir que se tiene un futuro. Este factor tiene mayor probabilidad de desarrollarse, en la medida que el AM realice una evaluación satisfactoria de lo vivido.

Al tomar en cuenta los hallazgos de esta investigación, se puede concluir que los factores comunes relevantes para la evaluación de la CV en el AM serían el apoyo social y la autoaceptación, elementos importantes de incluir en los programas de intervención gubernamentales como no gubernamentales destinados a favorecer la CV del AM.

A futuro, es importante para investigaciones sobre factores vinculados con $\mathrm{CV}$, tomar ciertas precauciones relacionadas con que las formas de medición sean sensibles a recoger aspectos relacionados con el género o que consideren aspectos evolutivos, como las teorías sobre el ciclo vital del $\mathrm{AM}^{32}$ y otros aspectos de la vida, como la espiritualidad.

Es necesario considerar que, tal como se ha mencionado, la muestra fue obtenida fundamentalmente en grupos organizados de adultos mayores, lo que podría explicar la positiva valoración e importancia del dominio participación social, siendo esta la única variable cuya media fue significativamente distinta de la de aquellos adultos mayores que no pertenecían a agrupaciones. En este mismo marco, es sabido que el mayor porcentaje de adultos que participan en agrupaciones son mujeres, lo que también puede estar implicado en que la participación social también fue la única variable en la que se encontró una diferencia estadísticamente significativa, corroborando el hecho de la importancia que adquiere para las mujeres la incorporación a grupos organizados, constituyéndose en una gran fuente de apoyo social. Dado este fenómeno, es que se hace necesario que futuras investigaciones profundicen en aspectos vinculados a la ausencia total de apoyo social tales como la condición de abandono y aislamiento, o la no participación en grupos organizados, fundamentalmente en hombres, ya que se trataría de personas con distintas motivaciones vitales que quienes acceden a estos centros, pudiendo abrir nuevas discusiones en el tema de CV y la autopercepción de salud. Es 
importante igualmente considerar en próximos estudios las preferencias subjetivas del adulto mayor en relación a las dimensiones vinculadas a $\mathrm{CV}$, a la vez que realizar estudios longitudinales de CV con el objetivo de evaluar posibles cambios durante el proceso de envejecimiento.

\section{Referencias}

1. Instituto Nacional de Estadísticas. INE. Estadísticas vitales 2007, Informe anual 2007. Santiago: Chile, 2009. Disponible en http://www.ine.cl/canales/chile_ estadistico/demografia_y_vitales/estadisticas_vitales/2010/04_01_10/vitales2007.pdf. [Consultado el 22 de noviembre de 2010].

2. Servicio Nacional del Adulto Mayor. SENAMA. Las Personas Mayores en Chile: Situación, avances y desafíos del envejecimiento y la vejez. Gobierno de Chile, Servicio Nacional del Adulto Mayor. Santiago, Chile. 2009.

3. Comisión Económica Para América Latina y El Caribe. CEPAL. Análisis comparativo sobre los programas para adultos mayores en México. División de Desarrollo Social, Santiago, Chile. 2010; 161.

4. Azpiazu M, Cruz A, Villagrasa J, Abanades J, García N, Alvear F. Factores asociados a mal estado de salud percibido o a mala calidad de vida en personas mayores de 65 años. Revista Española de Salud Pública 2002; 76: 683-99.

5. Mora M, Villalobos D, Araya G, Ozols A. Perspectiva subjetiva de la calidad de vida del adulto mayor, diferencias ligadas al género y a la práctica de la actividad físico recreativa. Revista MH Salud 2004; 1: 1-11.

6. Molzahn A, Skevington S, Kalfoss M, Makaroff K. The importance of facets of quality of to older adults: an international investigation. Quality of Life Research 2010; 19: 293-8

7. Medina M, CarbonelL M . Evaluación de la calidad de vida de las personas mayores en el municipio de Murcia. Portularia 2004; 4: 179-86.

8. Linsey H, Byrne R. Older people's views advance knowledge and measurement of quality of lfe and contribute to concepts of ageing well. Australian Association of Occupational Therapists 2007; 54: 162-3.

9. Torres M, Quezada M, Rioseco R, Ducci M. Calidad de vida de adultos mayores pobres de viviendas básicas: Estudio comparativo mediante uso de WHOQOL-BREF. Rev Med Chile 2008; 136: 325-33.

10. Vasconcelos G, Araújo L, Araújo L, Henrique M. Qualidade de vida e fatores associados em idosos dependentes em uma cidade do interior do Nordeste, Jornal Brasileiro de Psiquiatria 2009; 58: 39-44.

11. Araníbar P. Calidad de vida y vejez. Disponible en sitio Web del Centro interdisciplinar de Assitência e pesquisa em envelhecimento: www.ciape.org.br/. [Consultado el 20 de octubre de 2010].

12. Organización Mundial de La Salud. OMS . Boletín sobre el envejecimiento: perfiles y tendencias. Departamento de promoción de la salud, prevención y vigilancia de las enfermedades no contagiosas 2001; (4-5): 3-32.

13. Roses M. Calidad de vida y longevidad: un nuevo reto para la salud pública en las Américas. Revista Panamericana de Salud Pública 2005; 17: 295-6.

14. Sarabia C. Envejecimiento exitoso y calidad de vida. Su papel en las teorías del envejecimiento. Revista de la Sociedad Española de Enfermería Geriátrica y Gerontológica 2009; 20: 172-4.

15. WHOQOL Group. The World Health Organization Quality of Life Assessment (WHOQOL). Position Paper from the World Health Organization. Soc Sci Med 1995; 41: 1403-9.

16. Navarro E, Meléndez J, Tomás J. Relaciones entre variables físicas y de bienestar en la calidad de vida de las personas mayores, Revista de la Sociedad Española de Enfermería Geriátrica y Gerontológica 2008; 19: 63-7.

17. Ortiz J, Castro M. Bienestar psicológico de los adultos mayores, su relación con la autoestima y la autoeficacia. Contribución de enfermería. Ciencia y enfermería 2009; 15: 25-31.

18. Cardona D, Estrada A, Agudelo H. Calidad de vida y condición de salud de la población adulta de Medellín. Revista Biomédica del Instituto Nacional de Salud 2005; 26: 206-15.

19. Inga J, Vara A. Factores asociados a la satisfacción de vida de adultos mayores de 60 años en Lima- Perú. Univ. Psych 2006; 5: 475-85.

20. Fleck M, Chachamovich E, Trentini C. Development and validation of the portugueses version of the WHOQOLOLD module, Revista Saúde Pública 2006; 40: 793-9.

21. Power M, Quinn K, Schmidt S, The WHOQOL-OLD Group. Development of the WHOQOL-OLD module. Quality of Life Research 2005; 14: 2197-14.

22. Lucas R, Monteserín R, Juncà S. Quality of life and socio-demographic characteristics of older adults (the WHOQOL-OLD project). España, Barcelona: Universidad Autónoma de Barcelona, Instituto Catalán del Envejecimiento. 2007.

23. Díaz D, Rodríguez-Carvajal R, Blanco A, Moreno-Jiménez B, Gallardo I, Valle C, et al. Adaptación española de las escalas de bienestar psicológico de Ryff. Psicothema 2006; 18: 572-7.

24. Piña J, Rivera B. Validación del cuestionario de apoyo 
social funcional en personas seropositivas al VIH del noroeste de México. Ciencia y Enfermería 2007; 13: 5363.

25. Ware Jr, Kosinski M, Keller SD. A 12-Item Short-Form Health Survey: construction of scales and preliminary tests of reliability and validity. Medical Care 1996; 34: 220-33.

26. Cifre E, Salanova M. Validación factorial del "General Health Questionnaire” (GHQ-12) Mediante un análisis factorial confirmatorio, Revista de Psicología de la salud 2000; 12: 75-89.

27. Avlund K, Lund R, Holstein B, Due P. Social relations as determinant of onset of disability in aging. Archives of Gerontology and Geriatrics 2003; 38: 85-99.

28. Grammatikopoulos I, Koutentakis C. Social activity and participation as determinants of anxiety and depression among elderly in primary care. Annals of general psychiatry 2010; 9: 137.

29. Urzúa A, Jarne A. Calidad de Vida y Factores Biopsicosociales en Patologías Médicas Crónicas. Terapia Psicológica 2008; 26: 207-14.

30. Gallegos K, García C, Durán C, Reyes H, Durán L. Autopercepción del estado de salud: una aproximación a los ancianos en México, Revista Salud Pública 2006; 40: 792-801.

31. Sanjuán P, Pérez A, Bermúdez J. Escala de Autoeficacia General: Datos Psicométricos de la adaptación para población Española. Psicothema 2000; 12: 509-13.

32. Villar F, Triadó C, Resano C, Osuna M. Bienestar, adaptación y envejecimiento: cuando la estabilidad significa cambio. Mult Gerontol 2003; 13: 152-62.

33. Krzeimen D, Urquijo S, Monchietti A. Aprendizaje social. Estrategias de afrontamiento a los sucesos críticos del envejecimiento femenino. Psicothema 2004; 16: 350-6.

34. Gómez V, Villegas De Posada C, Barrera F, Cruz J. Factores predictores de bienestar subjetivo en una muestra colombiana. Rev Latinoamericana de Psicología 2007;
39: 311-25.

35. Matsuo M, Nagasawa J, Yoshino A, Hiromatsu K, Kurashiki K. Effects of Activity Participation of the Elderly on Quality of Life. Yonago Acta Médica 2003; 46: 17-24.

36. Vera M. Significado de calidad de vida del adulto mayor para sí mismo y para su familia. An Fac Med Lima 2007; 68: 284-90.

37. Osorio P, Torrejón M, Vogel N. Aproximación a un concepto de calidad de vida en la vejez. Revista de Psicología 2008; XVII: 101-8.

38. Oramas A, Santana S, Vergara A, El bienestar psicológico, un indicador positivo de la salud mental. Revista Cubana de Salud y Trabajo 2006; 7: 34-9.

39. Puga D, Rosero L, Glaser K, Castro T. Red social del adulto mayor en perspectiva comparada: Costa Rica, España e Inglaterra. Población y Salud en Mesoamerica 2007; 5: 1-21.

40. Arredondo A, Márquez E, Moreno F, Bazán M. Influencia del apoyo social en el control del paciente diabético tipo 2. Revista de Especialidades Médico-Quirúrgicas 2006; 11: 43-8.

41. Lemos S, Fernández J. Redes de soporte social y salud. Psicothema 1990; 2: 113-35.

42. Castellón A, Romero V. Autopercepción de la calidad de vida. Mult Gerontol 2004; 14: 131-7.

43. Fernández C, Oliva A. Social support, Psychological, Well- Being and health amongo the elderly. Educational Gerontology 2007; 33: 1053-68.

44. Cadenas R, Villareal E, Vargas E, Martínez L, Galicia L. Relación entre apoyo social funcional y satisfacción del adulto mayor jubilado. Revista Médica del Instituto Mexicano del Seguro Social 2009; 47: 311-4.

45. Martí J, Martínez F, Martí M, Marí R. Responsabilidad Social Universitaria: acción aplicada de valoración del bienestar psicológico en personas adultas mayores institucionalizadas. Revista de la Universidad Bolivariana 2007; 18: 1-13. 